\title{
Assessment of the availability and use of maternal health supplies in the primary health care system in Amhara Region, Ethiopia
}

Sarah Raifman

Sisay Mellese

Kelemua Hailemariam

Ian Askew

Population Council

Annabel Erulkar

Population Council

Follow this and additional works at: https://knowledgecommons.popcouncil.org/departments_sbsr-rh

Part of the Demography, Population, and Ecology Commons, Family, Life Course, and Society Commons, Gender and Sexuality Commons, International Public Health Commons, Maternal and Child Health Commons, Sociology of Culture Commons, and the Women's Health Commons How does access to this work benefit you? Let us know!

\section{Recommended Citation}

Raifman, Sarah, Sisay Mellese, Kelemua Hailemariam, lan Askew, and Annabel Erulkar. 2013. "Assessment of the availability and use of maternal health supplies in the primary health care system in Amhara Region, Ethiopia," Final Report. Addis Ababa: Population Council. 


\section{ASSESSMENT OF THE AVAILABILITY AND USE OF MATERNAL HEALTH SUPPLIES}

IN THE PRIMARY HEALTH CARE SYSTEM IN AMHARA REGION, ETHIOPIA

\section{FINAL REPORT}

SARAH RAIFMAN, SISAY MELLESE, KELEMUA HAILEMARIAM, IAN ASKEW and ANNABEL ERULKAR 


\section{(P) Population Council}

Population Council confronts critical health and development issues-from stopping the spread of HIV to improving reproductive health and ensuring that young people lead full and productive lives. Through biomedical, social science, and public health research in 50 countries, we work with our partners to deliver solutions that lead to more effective policies, programs, and technologies that improve lives around the world. Established in 1952 and headquartered in New York, the Council is a nongovernmental, nonprofit organization governed by an international board of trustees.

Population Council

Heritage Plaza, Fourth Floor

Post Office Box 25562-1000

Addis Ababa, Ethiopia

Tel: +12123390500

Fax: +12127556052

popcouncil.org

Suggested citation: Raifman, S., S. Mellese, K. Hailemariam, I. Askew and A. Erulkar. 2013. "Assessment of the Availability and Use of Maternal Health Supplies in the Primary Health Care System in Amhara Region, Ethiopia." Addis Ababa: Population Council. 


\section{ACKNOWLEDGMENTS}

Population Council gratefully acknowledges the support of the John D. and Catherine T. MacArthur Foundation for its continuing support for programs in the Council's Reproductive Health division and more specifically for its funding of this assessment of maternal health needs in the Amhara region of Ethiopia.

Population Council is indebted to Ethiopia's Federal Ministry of Health and Amhara region officials who provided assistance and recommendations for this study. We also would like to express our sincere appreciation to the staff and patrons of the woreda health facilities where this assessment was conducted. 



\section{TABLE OF CONTENTS}

Introduction......................................................................................

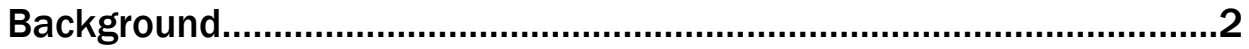

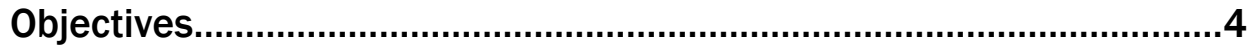

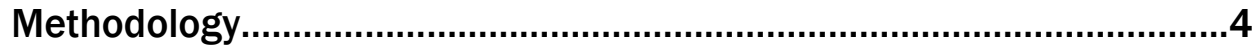

Selection of Health Facilities for Assessment............................4

Data Collection............................................................................5

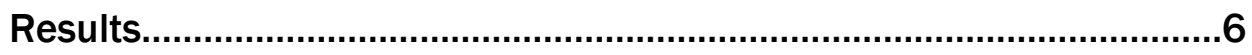

Facility Characteristics and Infrastructure..............................6

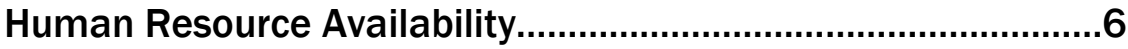

Maternal Health Service Availability.............................................6

Basic Emergency Obstetric and Neonatal Care (EmONC)

Service Availability.....................................................................

Safe and Clean Delivery Conditions.........................................8

Equipment, Materials, and Medical Devices for Clean and Safe Delivery and EmONC Services Availability....................................8

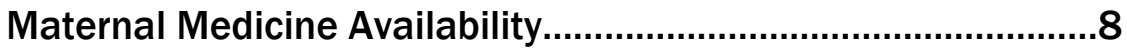

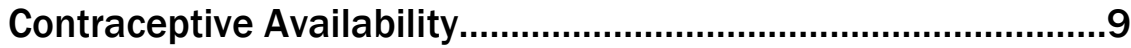

Guidelines and Protocols...............................................................

Provider Knowledge, Training, and Experience........................10

Supply Chain Management System.......................................13

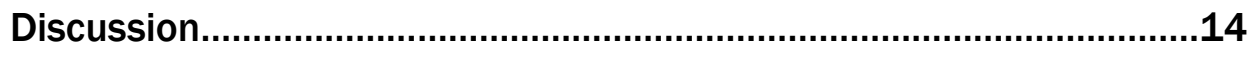

Availability of Maternal Health Supplies................................14

Needs, Gaps, and Challenges......................................................14

Readiness for Integrating Misoprostol into HEP......................15

Overall Recommendations.....................................................15

Dissemination.......................................................................17

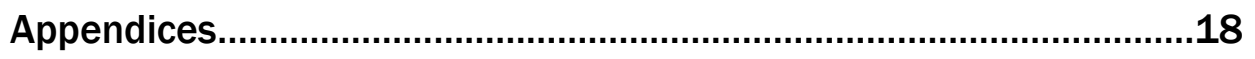

References....................................................................................25 


\section{List of Abbreviations}

\begin{tabular}{|c|c|}
\hline ANC & Antenatal care \\
\hline ART & Antiretroviral therapy \\
\hline BEmONC & Basic emergency obstetric and neonatal care \\
\hline CEmONC & Comprehensive emergency obstetric and neonatal care \\
\hline CHV & Community health volunteer \\
\hline EmONC & Emergency obstetric and neonatal care \\
\hline EDHS & Ethiopia demographic and health survey \\
\hline EML & Essential medicines list \\
\hline FP & Family planning \\
\hline FMoH & Federal Ministry of Health \\
\hline $\mathrm{HC}$ & Health center \\
\hline HEP & Health extension program \\
\hline HEW & Health extension worker \\
\hline HMIS & Health management information system \\
\hline HP & Health post \\
\hline HSDP & Ethiopia's health sector development plans \\
\hline iCCM & Integrated community case management \\
\hline IMNCH & Integrated management of neonatal and childhood illnesses \\
\hline IPLS & Integrated pharmaceuticals logistics system \\
\hline IU & International units \\
\hline MDG & Millennium development goal \\
\hline $\mathrm{MgSO}_{4}$ & Magnesium sulfate \\
\hline $\mathrm{MNCH}$ & Maternal neonatal and child health \\
\hline PAC & Postabortion care \\
\hline $\mathrm{PE} / \mathrm{E}$ & Pre-eclampsia/eclampsia \\
\hline PFSA & Pharmaceuticals fund \& supply agency \\
\hline РМтСТ & Prevention of mother-to-child transmission \\
\hline PNC & Postnatal care \\
\hline PPH & Postpartum hemorrhage \\
\hline RH & Reproductive health \\
\hline $\mathrm{RH} / \mathrm{FP}$ & Reproductive health/family planning \\
\hline SDP & Service delivery point \\
\hline SRS & Simple random sampling \\
\hline STI & Sexually transmitted infection \\
\hline TFR & Total fertility rate \\
\hline VCT & Voluntary counseling and testing \\
\hline VDRL & Venereal disease research laboratory \\
\hline
\end{tabular}




\section{INTRODUCTION}

Maternal mortality in Ethiopia remains among the highest in the world (Hogan et al. 2010). Ethiopia is among the six high burden countries for maternal death, along with Afghanistan, the Democratic Republic the Congo (DRC), India, Nigeria and Pakistan, which collectively account for roughly 50 percent of all maternal deaths worldwide. With a current estimate of 676 maternal deaths per 100,000 live births, it is unlikely that Ethiopia will achieve its Millennium Development Goal (MDG) 5 target of reducing the maternal mortality ratio by three quarters by 2015 (EDHS 2011, Hogan et al. 2010). The leading causes of maternal death in Ethiopia are postpartum hemorrhage (PPH), unsafe abortion, infection, pregnancy related hypertension, and obstructed labor (Koblinsky et al. 2010). Ensuring availability of modern contraceptives and essential life-saving maternal/RH medicines is a major challenge in Ethiopia, where unmet need is very high and funding for supplies is almost completely donor dependent (Morrison and Brundage 2012).

A UN Commission Report in 2012 identified 13 life-saving commodities, defined as medicines, medical devices, and health supplies that effectively address avoidable causes of death during pregnancy and childbirth that, if more widely accessed and properly used, could significantly reduce preventable deaths among women (Table 1) (UN Commission Report 2012).

This assessment evaluates the availability of life-saving maternal medicines (oxytocin, misoprostol, $\mathrm{MgSO}_{4}$ and antibiotics), equipment, medical devices, and materials that are essential for basic emergency obstetric and neonatal care (BEmONC) and safe and clean delivery services within primary health care in Ethiopia.

Table 1: Life-saving commodities within the continuum of care

\begin{tabular}{|l|l|l|l|}
\hline Reproductive health & Maternal health & Newborn health & Child health \\
\hline $\begin{array}{l}\text { Female condom: } \\
\text { Prevents HIV and } \\
\text { unintended pregnancy }\end{array}$ & $\begin{array}{l}\text { Oxytocin: } \\
\text { Prevents and treats } \\
\text { PPH }\end{array}$ & $\begin{array}{l}\text { Injectable antibiotics: } \\
\text { Treats newborn sepsis }\end{array}$ & $\begin{array}{l}\text { Amoxicillin: } \\
\text { Treats } \\
\text { Pneumonia }\end{array}$ \\
\hline $\begin{array}{l}\text { Contraceptive implants: } \\
\text { Prevents unintended } \\
\text { pregnancy }\end{array}$ & $\begin{array}{l}\text { Misoprostol: } \\
\text { Prevents and treats } \\
\text { PPH }\end{array}$ & $\begin{array}{l}\text { Antenatal corticosteroids: } \\
\text { Prevents preterm } \\
\text { respiratory distress } \\
\text { syndrome }\end{array}$ & $\begin{array}{l}\text { Oral rehydration } \\
\text { salt: } \\
\text { Prevents } \\
\text { dehydration from } \\
\text { diarrhea }\end{array}$ \\
\hline $\begin{array}{l}\text { Emergency contraceptive: } \\
\text { Prevents unintended } \\
\text { pregnancy }\end{array}$ & $\begin{array}{l}\text { MgSO4: } \\
\text { Prevents and treats } \\
\text { pre-eclampsia and } \\
\text { eclampsia }\end{array}$ & $\begin{array}{l}\text { Chlorhexidine: } \\
\text { Prevents umbilical cord } \\
\text { infection }\end{array}$ & $\begin{array}{l}\text { Zinc: } \\
\text { Treats diarrhea }\end{array}$ \\
\hline & & $\begin{array}{l}\text { Resuscitation devices: } \\
\text { For newborn asphyxia }\end{array}$ & \\
\hline
\end{tabular}

Source: UN Commission Report 2012 


\section{Figure 1: Structure of Ethiopia's health system}

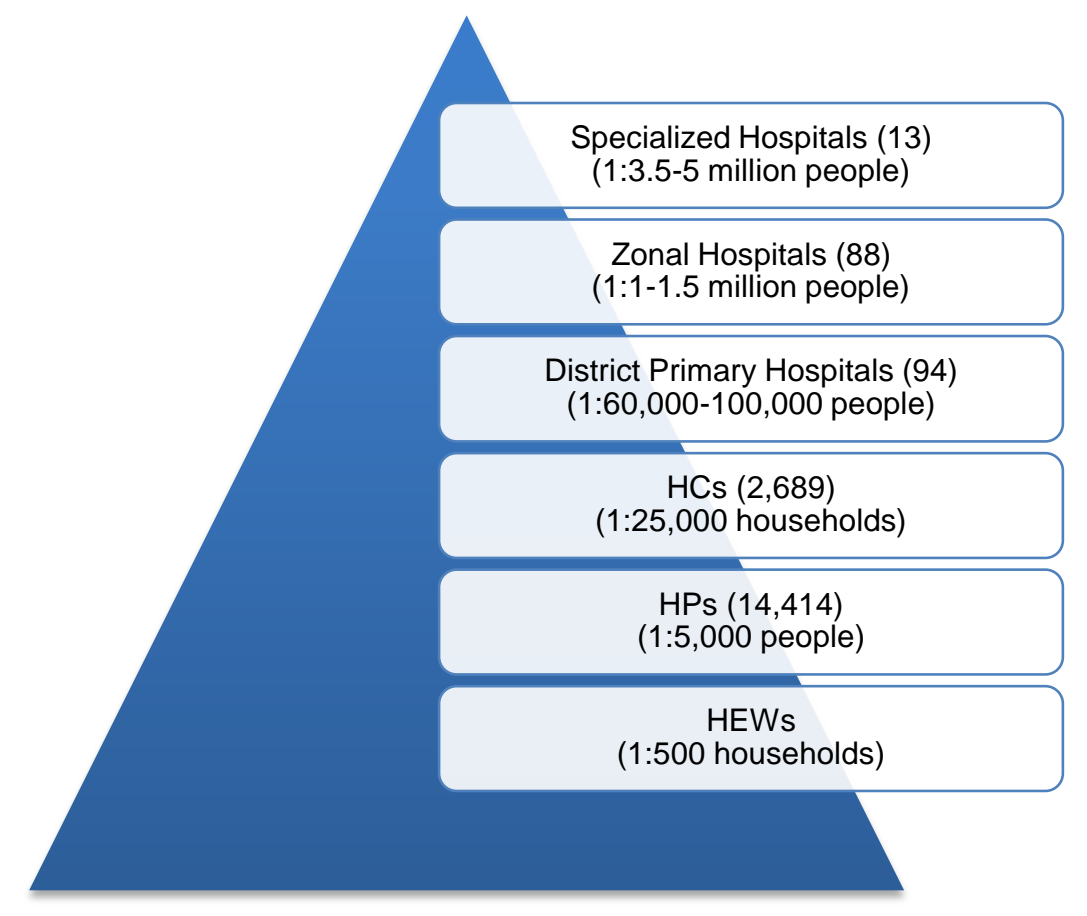

Source: FMoH, HSDP IV 2010-2011, 2014-2015, October 2010

The government is the primary health care provider in Ethiopia. The country's health system is decentralized and organized into three tiers connected by a referral system (Figure 1). Health centers (HCs) serve as the first curative referral center from health posts (HPs) for services not provided at the HP level, such as basic and comprehensive EmONC. HPs are expected to provide clean and safe delivery services, basic reproductive health (RH) services (family planning, antenatal care, postnatal care), and immunization services. HCs are expected to provide emergency care in addition to safe and clean delivery and basic RH services (Raifman et al. 2012). Since 1997, Ethiopia's Health Sector Development Plans (HSDP), a 20 year strategy of four consecutive, five year investment programs, have aimed to increase the number of providers and health care facilities, as well as further develop preventive and curative health care components, and promote private and NGO participation in the health sector. One of the most ambitious components of the HSDP was the creation in 2004 of the rural Health Extension Program (HEP). The Federal Ministry of Health (FMoH) launched HEP to make essential healthcare universal, through community provision of preventive and limited curative and rehabilitative services (FMoH 2004, Mekbib 2007). In the last decade, the Government of Ethiopia has been aggressively increasing service coverage in rural areas by constructing additional service delivery points (from 6191 HPs in 2004-05 to 14,416 in 2009-10) and deploying Health Extension Workers (HEWs), from 2,737 in 2004 to 34,382 in 2010, as well as community health volunteers (CHVs) at the community level (FMoH 2010).

The Health Policy and Health Sector Development Plan makes provisions for RH and emphasizes the importance of access to essential drugs and distribution of contraceptives and commodities at service delivery points (SDPs). The Plan acknowledges current challenges in supply, including shortages of skilled midwives, a weak referral system among HCs, lack of adequate EmONC equipment availability, and under-financing for health services (HSDP-IV 2010-11, 2014-15). 


\section{BACKGROUND}

The most effective medicines used to prevent postpartum hemorrhage (PPH) - the leading cause of maternal death in Ethiopia and worldwide - are oxytocin and misoprostol (WHO 2009, Central Statistical Agency et al. 2008). Oxytocin is recommended by the World Health Organization (WHO) as the first line drug for active management of the third stage of labor (AMTSL) 1 and management of excessive bleeding after childbirth. Oxytocin is effective sooner than other uterotonic drugs and is widely produced by more than 100 manufacturers globally (MSH 2011), but oxytocin is temperature sensitive and relatively expensive (US $\$ 0.18$ for $10 \mathrm{IU}$ ) (PATH 2008). Misoprostol can be stored at room temperature and is inexpensive; therefore, in low resource settings, where oxytocin is not often available, misoprostol is the preferred method for $\mathrm{PPH}$ prevention. Among several indications for misoprostol use, however, abortion induction is the indication that most concerns many people. In some countries, there is a fear that oxytocin is used to hasten labor and is thus dangerous, but rates of induction and augmentation are relatively low in Ethiopia (POPPHI 2008).

For preventing and treating pre-eclampsia and eclampsia $(\mathrm{PE} / \mathrm{E})$, studies show that magnesium sulfate $\left(\mathrm{MgSO}_{4}\right)$ is the most effective first line drug of choice (WHO 2011). $\mathrm{MgSO}_{4}$ is administered by intravenous or intramuscular injection, requiring as many as nine vials for one treatment and costing US $\$ 0.10$ per mL, and it is produced by only one global manufacturer and many local manufacturers. Calcium gluconate, a mineral supplement, is an important antidote to $\mathrm{MgSO}_{4}$ when serious toxicity is suspected (Smith et al. 2013).

Oxytocin, misoprostol, and $\mathrm{MgSO}_{4}$ are all included in the $18^{\text {th }}$ edition of the WHO Model Lists of Essential Medicines (EML). In Ethiopia, oxytocin is included on the Essential Drug List for PPH prevention and treatment, and misoprostol is approved for prevention of $\mathrm{PPH}$ where oxytocin is not available. $\mathrm{MgSO}_{4}(500$ $\mathrm{mg} / \mathrm{mL}$ in $2 \mathrm{~mL}$ ampoule; $500 \mathrm{mg} / \mathrm{mL}$ in $10 \mathrm{~mL}$ ampoule) is included on the WHO EML for severe PE/E and has been approved for $\mathrm{PE} / \mathrm{E}$ within Ethiopia national policy. $\mathrm{MgSO}_{4}$ is also currently on the Ethiopian Essential Drug List and authorized for delivery by midwives, health officers, and clinical nurses at HCs (but only the loading dose) (FMoH 2012).

Six signal functions are essential for basic emergency obstetric and neonatal care BEmONC: administration of parenteral antibiotics, oxytocic drugs, anticonvulsants for PE/E, manual removal of placenta, removal of retained placenta, and assisted vaginal delivery (UNFPA, WHO, UNICEF 1997). Two additional signal functions-safe blood transfusions and surgery (i.e. cesarean delivery) - constitute the package of eight essential functions for comprehensive EmONC (CEmONC). More recently, neonatal resuscitation has also been included in BEmONC. If a facility performs all of the six or seven basic functions routinely, it qualifies as a BEmONC facility, and if a facility performs all eight or nine functions routinely, it qualifies a CEmONC facility. In Ethiopia, BEmONC is expected to be routine at the $\mathrm{HC}$ level and to perform these functions a facility must have the necessary life-saving equipment, materials, and supplies (UNICEF and FMoH 2004).

In Ethiopia, all public health facilities obtain essential and vital pharmaceuticals primarily through the integrated pharmaceuticals logistics system (IPLS), a single reporting and distribution system based on the overall mandate and scope of the national Pharmaceuticals Fund and Supply Agency. To be successful in ensuring that patients always receive pharmaceuticals, IPLS must fulfill the Six Rights (the right products, in the right quality, of the right quantity, at the right place, at the right time, and for the right cost). Among the pharmaceuticals managed by IPLS are $\mathrm{MCH}$ products, laboratory products, nutrition products, and environmental health and sanitation products (FMoH 2011).

\footnotetext{
1 AMTSL consists of three basic procedures completed by a skilled birth attendant: administration of a uterotonic drug within one minute following the birth, delivery of the placenta with controlled cord traction, and massage of the uterus after the placenta delivers (POPPHI 2007).
} 
Population Council is supporting the Amhara Bureau of Health to understand gaps in provision of maternal health services to ultimately accelerate Ethiopia's progress towards meeting MDG 5. This study assesses the availability and use of life saving maternal health medicines and equipment at HPs and HCs in Amhara.

\section{OBJECTIVES}

The main purpose of this study was to assess the availability and use of supplies and equipment for clean and safe delivery and EmONC services in Amhara region's primary healthcare service delivery points (SDPs). Specifically, this assessment provides current information on the needs, gaps, and challenges regarding misoprostol availability and use in HPs as well as $\mathrm{MgSO}_{4}$ and oxytocin availability and use in HCs. The availability of other related products, medical devices, materials and equipment critical for maternal health services were also assessed, as well as the availability of human resources required for skilled care.

The specific objectives of the assessment are:

1. To examine the availability of maternal health supplies at HPs and HCs in the selected communities;

2. To identify needs, gaps, and challenges that will inform program managers' strengthening of the primary health care system with increased availability of maternal health supplies;

3. To determine Amhara Bureau of Health's readiness for integrating the provision of misoprostol in its health extension programs;

4. To propose additional strategies for increasing access to maternal health supplies within HPs and HCs.

\section{METHODOLOGY}

\section{Selection of health facilities for assessment}

The assessment was conducted in 10 woredas (districts) in Amhara region's North Gondar and West Gojam zones. Amhara's population of over 17 million comprises almost one quarter of Ethiopia's population (UNFPA 2008). The large majority of Amhara's population lives in rural areas (87\%) and is Orthodox Christian. Amhara has shown consistently strong reproductive health and family planning (RH/FP) health indicators compared to other predominantly rural states (EDHS 2005, EDHS 2011). Amhara's total fertility rate (TFR) is 4.2, and total FP demand is slightly above the national average of 56 percent (EDHS 2011). Considerably more currently married women in Amhara (34\%) use a contraceptive than the national average $(29 \%)$, yet antenatal care (ANC) is still low, at 34 percent from any skilled provider and only seven percent from HEWs (EDHS 2011).

The sample frame for selecting facilities included all HPs and HCs in the study woredas (or districts), which provide maternal health services. Woredas were selected in consultation with Amhara district officials. These woredas have been the focus of ongoing health system strengthening activities, including the Council's Meserete Hiwott program. In Meserete Hiwott's original design, mentors of married girls distributed misoprostol, but later it focused on strengthening the health system to deliver misoprostol in the same woredas as the original mentoring program. As a result, district health officials wanted to obtain a descriptive picture of their district's readiness for delivering full maternal health services. Woreda health offices provided lists of facilities from which a sample of HCs and HPs were selected through simple random sampling (SRS), with the goal of selecting approximately two HPs for every one HC, and at least 30 percent of all facilities. Permission for the health facility assessments was obtained from the Bureau of Health and Health Departments of North Gondar and West Gojam zones. At the facilities, detailed information, including the assessment's purpose 
and procedure, its benefits, the voluntary nature of participation, and right to stop interviews at any time, was explained to respondents prior to obtaining informed consent. Interviewees were informed that participation was anonymous and confidential.

\section{Data collection}

A team of professionals from the Amhara Bureau of Health, the zonal Health Departments, and Population Council coordinated data collection. The team recruited medically trained data collectors, including one health officer, one midwife, one pharmacy technician, and three nurses, who received a five day training that included the assessment's purpose and data collection tool administration. After training, data collectors were organized into three teams, with two data collectors at each SDP. Depending on facility accessibility and information availability, each data collection team spent one full day at each SDP for inventories and health care provider interviews. The assessment team, led by the Council health care provider, worked closely with the data collectors and provided regular recommendations. The team visited the study facilities to check for accuracy and completeness of information collected.

Three data collection tools were employed:

\section{1) Inventory of maternal health supplies and services}

All maternal health supplies (medicines, medical devices, materials and equipment) from the selected HPs and HCs were listed by observing available items and through discussions with providers in charge of maternal health care services and facility pharmacies. In addition, data on services (ANC, delivery, PNC, and EmONC) provided within the 12 months preceding the survey were gathered from clinical records, registration books, or the health management information system (HMIS). Data on supply chain management issues, including logistics, stock outs and HMIS performance, were assessed.

\section{2) Healthcare provider interview}

All healthcare providers responsible for providing maternal health care services (including ANC, delivery, and PNC and EmONC) were interviewed using structured and unstructured questionnaires. Providers were expected to comprise midwives, nurses, health officers, medical doctors, pharmacy technicians, supply chain managers, and facility heads at HCs. In addition, one of the two HEWs assigned to each HP were interviewed for insight into their maternal health service practices, particularly misoprostol for $\mathrm{PPH}$ and safe and clean delivery services. Health care provider interviews addressed policy and program issues, including maternal health supply availability, provider training, knowledge, and skills and practices related to the administration of medicine and EmONC services. Furthermore, the availability of health care providers who can provide basic packages of EmONC at the HC level was assessed.

3) Key informant interviews (KIIs)

The assessment included key informant interviews with policymakers, program managers, and supply chain managers at woreda, zone, and regional Bureau of Health levels. The goal of KIIs was to generate information to influence regional policy on maternal health supply availability in the primary health care system and barriers to availability and provision of care. The KII interview guides included policy and programmatic issues such as registration of supplies in country, their inclusion on the essential drug list, provider authorization for administration of misoprostol, oxytocin and $\mathrm{MgSO}_{4}$ at each health facility level, plans for integrating misoprostol in the Health Extension Program, and treatment guidelines on maternal supplies administration.

Data were cleaned, coded, and stored in a file using EPI INFO and then imported into SPSS version 15 for descriptive analysis. 


\section{RESULTS}

Inventory data were collected in August 2013 from assessments of $23 \mathrm{HCs}$ and $36 \mathrm{HPs}$, along with interviews with $26 \mathrm{HC}$ providers and $36 \mathrm{HEWs.} \mathrm{Eight} \mathrm{KIIs} \mathrm{were} \mathrm{also} \mathrm{conducted} \mathrm{with} \mathrm{health} \mathrm{managers} \mathrm{at} \mathrm{zonal} \mathrm{health}$ departments (2), woreda health offices (3), and the Amhara Bureau of Health (2), as well as a supply chain manager from PFSA. Twenty-eight selected facilities (11 HCs and $17 \mathrm{HPs}$ ) are in North Gondar and 32 (12 HCs and $19 \mathrm{HPs}$ ) are in West Gojam; a complete list of facilities is included in Appendix 1. All HP providers interviewed were female HEWs between the ages of 21 and 32. In HCs, three health officers, 18 midwifery nurses, and five nurses (with diplomas) were interviewed, of whom $10(38 \%)$ were male and $16(62 \%)$ were female, ranging in age from 22 to 37. Most of these providers worked in the maternal and child health unit $(92 \%)$, or less frequently, in the outpatient department $(8 \%)$. The delivery case management team is the maternal and child health unit, which provides ANC, delivery, PNC, newborn care, and FP.

\section{Facility characteristics and infrastructure}

All HPs and most (74\%) HCs in the assessment are rural. Most HCs have robust infrastructure, including electricity (78\%), working toilets (91\%), a landline telephone (52\%), mobile telephone service (87\%), and computers (78\%). Few HPs, however, have electricity (11.1\%) and none have access to a landline telephone, computers, or car, and most $(92 \%)$ do not have mobile telephone service access. All HPs and HCs do have access to an ambulance. Complete data on facility infrastructure are included in Appendix 2.

\section{Human resource availability}

Available human resources in the HPs were comprised of HEWs only, as expected. Most HCs employed health officers $(96 \%)$, midwives $(96 \%)$, and nurses $(100 \%)$, but only about half $(52 \%)$ of HCs had nurses with formal degrees. Seven $(19 \%)$ of the HPs assessed reported HEW shortages, while more than half of the HCs reported human resource shortages (Appendix 3).

\section{Maternal health service availability}

Most primary health care services were provided at all HCs (Table 2), but EmONC was not reported at all of them. All 36 HPs, immunization, FP, PNC, and referral services were available, and most indicated availability of ANC (97\%), safe delivery services (81\%), and rapid diagnostic tests for malaria (94\%). As expected, EmONC, safe abortion, postabortion care services, and HIV testing and counseling services were not available, as these services are not permitted within HPs (Table 3).

Table 2: Service availability, by health facility

\begin{tabular}{|l|c|c|}
\hline \multicolumn{1}{|c|}{ Type of service available } & $\begin{array}{c}\text { Health post } \\
\mathbf{n = 3 6}\end{array}$ & $\begin{array}{c}\text { Health center } \\
\mathbf{n = 2 3}\end{array}$ \\
\hline Family planning (FP) & $36(100 \%)$ & $23(100 \%)$ \\
\hline Antenatal care (ANC) & $35(97.2 \%)$ & $23(100 \%)$ \\
\hline Delivery & $29(80.6 \%)$ & $23(100 \%)$ \\
\hline Postnatal care (PNC) & $36(100 \%)$ & $23(100 \%)$ \\
\hline Emergency obstetric and neonatal care (EmONC) & $\mathrm{N} / \mathrm{A}$ & $18(78.3 \%)$ \\
\hline Safe abortion & $\mathrm{N} / \mathrm{A}$ & $10(43.5 \%)$ \\
\hline Postabortion care (PAC) & $\mathrm{N} / \mathrm{A}$ & $21(91.3 \%)$ \\
\hline Prevention of mother-to-child transmission (PMTCT) & $\mathrm{N} / \mathrm{A}$ & $20(87 \%)$ \\
\hline Immunization & $36(100 \%)$ & $23(100 \%)$ \\
\hline Antiretroviral therapy (ART) & $\mathrm{N} / \mathrm{A}$ & $7(30.4 \%)$ \\
\hline Voluntary counseling and testing (VCT) & $\mathrm{N} / \mathrm{A}$ & $21(91.3 \%)$ \\
\hline Sexually transmitted infections (STI) & $\mathrm{N} / \mathrm{A}$ & $23(100 \%)$ \\
\hline Nutrition & $36(100 \%)$ & $23(100 \%)$ \\
\hline Laboratory & $\mathrm{N} / \mathrm{A}$ & $23(100 \%)$ \\
\hline Referral & $36(100 \%)$ & $23(100 \%)$ \\
\hline
\end{tabular}


Table 3: Availability of tests, by facility type

\begin{tabular}{|l|c|c|}
\hline \multicolumn{1}{|c|}{ Tests Available } & $\begin{array}{c}\text { Health post } \\
\mathbf{n = 3 6}\end{array}$ & $\begin{array}{c}\text { Health center } \\
\mathbf{n = 2 3}\end{array}$ \\
\hline HIV testing & N/A & $22(95.7 \%)$ \\
\hline VDRL testing for syphilis & N/A & $8(34.8 \%)$ \\
\hline Blood test for anemia & N/A & $12(52.2 \%)$ \\
\hline Urine test for protein & N/A & $18(78.3 \%)$ \\
\hline Urine test for glucose & N/A & $15(65.2 \%)$ \\
\hline Blood test for malaria & $34(94.4)$ & $22(95.7 \%)$ \\
\hline
\end{tabular}

Source of Table 2 and Table 3: Inventory

\section{Basic Emergency Obstetric and Neonatal Care (EmONC) service availability}

The availability of all six functions of BEmONC was not universal in the HCs (Table 4, Figure 2). Oxytocin for $\mathrm{PPH}$ was virtually available at all HCs, and almost all had performed manual placenta removal in the previous 12 months. Less than one third of HCs had performed removal of retained products of conception using MVA, however, presumably due to lack of equipment or trained providers.

Table 4: EmONC service availability in health centers

\begin{tabular}{|l|c|}
\hline \multicolumn{1}{|c|}{$\begin{array}{c}\text { Were the following basic emergency obstetric care services performed at least once } \\
\text { during the last 12 months? }\end{array}$} & $\begin{array}{l}\text { Health center } \\
n=23\end{array}$ \\
\hline Parenteral antibiotics for maternal sepsis given in last 12 months? & $14(60.9 \%)$ \\
\hline Parenteral oxytocic for PPH given in last 12 months in this facility? & $\mathbf{2 3 ( 1 0 0 \% )}$ \\
\hline Parenteral anticonvulsant for PE/E given in last 12 months in this facility? & $22(95.7 \%)$ \\
\hline Manual removal of placenta performed in last 12 months at this facility? & $7(30.4 \%)$ \\
\hline Removal of retained products of conception performed using MVA in the last 12 months in this facility? & $11(47.8 \%)$ \\
\hline Assisted delivery performed in the last 12 months in this facility? & \\
\hline
\end{tabular}

Source: Healthcare provider interview

Figure 2: Total score of Basic Emergency Obstetric services (BEmONC) available at health centers

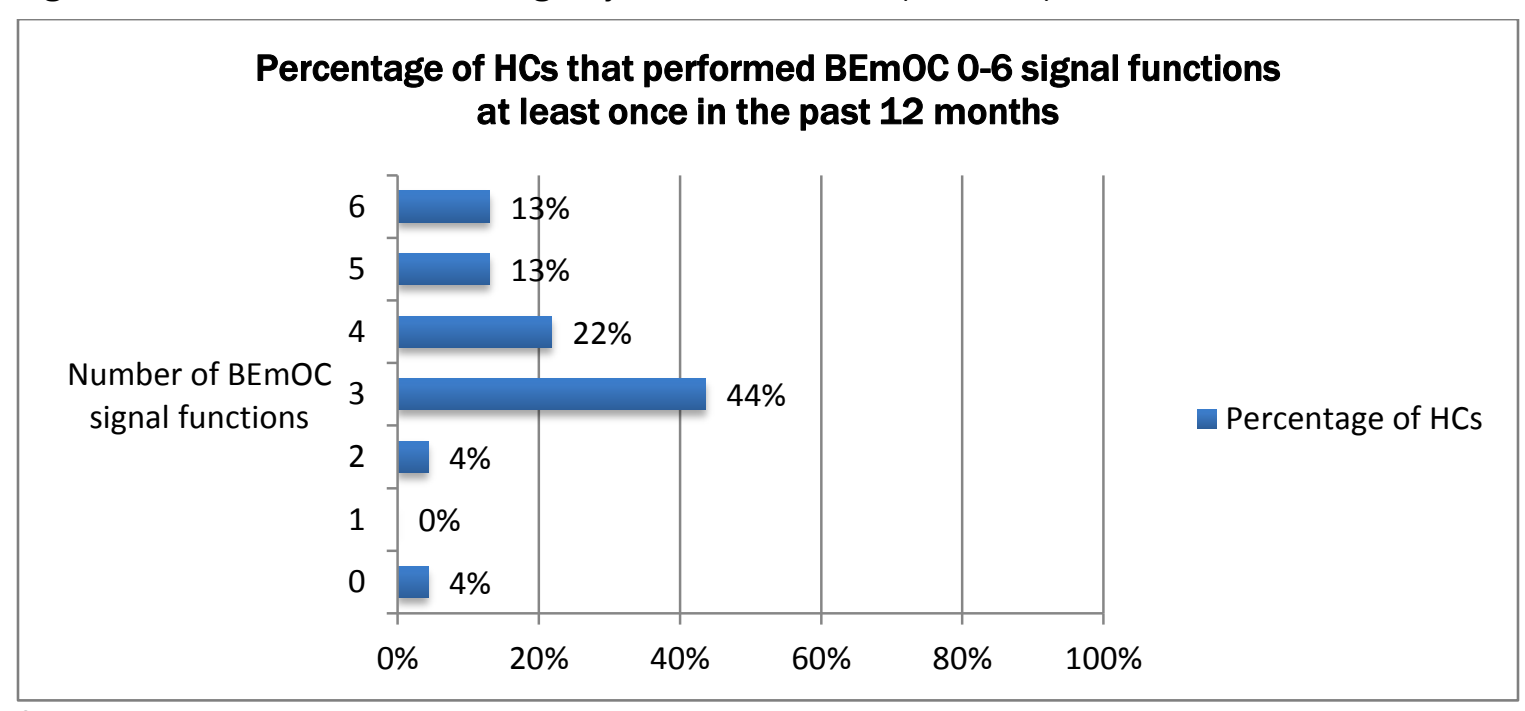

Source: Healthcare provider interview 


\section{Safe and clean delivery conditions}

Clean childbirth practices utilizing the "six cleans" (clean hands, clean surface, clean blade, clean cord tie clean towel to wrap baby, and clean cloth for mother) are important for averting maternal and neonatal infections, and are expected to be part of safe and clean delivery practices in HPs, where skilled birth attendants are virtually unavailable. The condition of the "six cleans" for safe and clean delivery services by facility is presented in Table 5. Although most HCs had access to the "six cleans," relatively few (35\%) had clean cloths or baby towels. In HPs, facilities were even less equipped with the "six cleans."

Table 5: "Six cleans" availability, by type of health facility

\begin{tabular}{|l|c|c|}
\hline \multicolumn{1}{|c|}{ Status of maternity rooms } & $\begin{array}{l}\text { Health post } \\
\mathrm{n=36}\end{array}$ & $\begin{array}{l}\text { Health center } \\
\mathrm{n}=23\end{array}$ \\
\hline Clean rooms/surface & $16(44 \%)$ & $23(100 \%)$ \\
\hline Clean bed sheet/rubber sheet & $16(44 \%)$ & $17(73.9 \%)$ \\
\hline Clean/sterile blade & $26(72.2 \%)$ & $23(100 \%)$ \\
\hline Clean cloth/baby towel for wrapping baby & $5(13.9 \%)$ & $8(34.8 \%)$ \\
\hline Clean/sterile cord tie & $28(72.8 \%)$ & $23(100 \%)$ \\
\hline Adequate water for washing & $16(44 \%)$ & $22(95.7 \%)$ \\
\hline Disinfectant/soap & $16(44 \%)$ & $22(95.7 \%)$ \\
\hline
\end{tabular}

Source: Inventory

\section{Equipment, materials, and medical devices for clean and safe delivery and EmONC services availability}

Equipment, material, and medical device availability is essential for clean and safe delivery in HPs and EmONC at HCs. The surveyed HPs had inadequate access to key equipment required for clean and safe delivery: Many HPs lacked torches, examination couches, resuscitation devices, syringes, and suturing material necessary for obstetric procedures. HCs were relatively better equipped, as expected, but many HCs also lacked access to torches, Ambubags, manual vacuum aspirators, and suturing material. See Appendix 4 for complete data on availability of equipment and materials for safe and clean delivery and EmONC services.

\section{Maternal Medicine availability}

Table 6 illustrates maternal medicine availability for HPs and HCs and the percentage of facilities with stock outs in the 12 months prior to the assessment. Oxytocin was available at all HCs, and only one facility reported stock outs within the last 12 months. Misoprostol for PPH prevention was available at nine $(39 \%)$ HCs for use in safe abortion services and at two (6\%) HPs. Only two (8\%) HCs, compared with $26(72 \%)$ HPs, reported stock outs of misoprostol in the last 12 months. $\mathrm{MgSO}_{4}$ was not present at any facility; and furthermore, all assessed HCs reported that the drug had never been available. Calcium gluconate, a common $\mathrm{MgSO}_{4}$ antidote, was available at only one (4\%) HCs. Complete data maternal medicine availability are included in Appendix 5.

Table 6: Availability and stock outs of key maternal medicines

\begin{tabular}{|l|c|c|}
\hline \multicolumn{1}{|c|}{ Available Maternal Medicines } & $\begin{array}{c}\text { Health Post } \\
\mathrm{n=36}\end{array}$ & $\begin{array}{c}\text { Health Center } \\
\mathrm{n}=\mathbf{2 3}\end{array}$ \\
\hline Oxytocin & $\mathrm{N} / \mathrm{A}$ & $\mathbf{2 3}(\mathbf{1 0 0 \% )}$ \\
\hline Misoprostol & $2(5.6 \%)$ & $9(39.1 \%)$ \\
\hline $\mathrm{MgSO}_{4}$ & $\mathrm{~N} / \mathrm{A}$ & $0(0 \%)$ \\
\hline Calcium gluconate & $\mathrm{N} / \mathrm{A}$ & $1(4.3 \%)$ \\
\hline Normal saline & N/A & $22(95.7 \%)$ \\
\hline Ringer lactate & N/A & $20(87 \%)$ \\
\hline
\end{tabular}




\begin{tabular}{|l|c|c|}
\hline \multicolumn{1}{|c|}{ Stock outs of maternal medicines } & $\begin{array}{c}\text { Health Post } \\
\mathbf{n}=36\end{array}$ & $\begin{array}{c}\text { Health Center } \\
\text { n=26 }\end{array}$ \\
\hline Is oxytocin regularly available at this facility? & N/A & $25(96.2 \%)$ \\
\hline $\begin{array}{l}\text { Stock outs of oxytocin during the last 12 } \\
\text { months? }\end{array}$ & N/A & $1(3.8 \%)$ \\
\hline Is misoprostol regularly available at this facility? & $2(5.6 \%)$ & $10(38.5 \%)$ \\
\hline Stock-outs of misoprostol in the last 12 & $26(72.2 \%)$ & $2(7.7 \%)$ \\
\hline Is MgSO4 regularly available at this facility? & N/A & 0 \\
\hline
\end{tabular}

Source: Inventory and healthcare provider interviews

\section{Contraceptive availability}

The most commonly used contraceptive in Ethiopia, Depo-provera, was available at $23(100 \%)$ HCs and 34 (94\%) HPs. Male condoms and combined pills were also available at most HCs and HPs. Progesterone-only pills were mostly available at HCs but not at HPs. Emergency contraception was available at roughly half of the HCs assessed, and only five (14\%) HPs. Implants were available to varying degrees, depending on type. See Appendix 6 for more detail.

\section{Guidelines and protocols}

Overall, the availability of guidelines, service protocols, and training manuals was poor at both HCs and HPs. Service protocols for PPH prevention and treatment with oxytocin were available at $11 \mathrm{HCs}$ and only two HPs. Most (66\%) HC providers reported knowing these guidelines "very well," but most HEWs (58\%) reported knowing them "not well," and 33 percent stated that the protocols did not even exist (Figure 3).

A training manual on safe and clean delivery, including information on misoprostol for $\mathrm{PPH}$, was available at few facilities (14\% of HPs and 17\% of HCs). When asked about misoprostol guidelines, 34 percent of HEWs reported "not [knowing them] well," and nearly 20 percent indicated that guidelines do not exist. A large majority of HC providers reported "not [knowing misoprostol guidelines] well" nor did they know whether misoprostol was being scaled up for provision in HPs in their respective districts (Figure 3). Three quarters of HP providers and one third of HC providers were aware that misoprostol had been piloted for home birth in their districts. And an even greater number of providers (81\% of HEWs and $38 \%$ of HC providers) reported that misoprostol was piloted at the HP level in their districts. Nevertheless, most HEWs interviewed said that misoprostol was not scaled up with either home birth or HP deliveries, and most HC providers did not know whether misoprostol was scaled up in their districts. Complete data on provider knowledge of expansion of misoprostol for PPH in the home is presented in Appendix 7.

A protocol for $\mathrm{MgSO}_{4}$ for PE/E was available at few HPs (8\%) and less than half of the HCs (48\%). Most HEWs (53\%) reported "not [knowing the PE/E protocol] well," and over 40 percent (42\%) did not know it existed. Most (70\%) HC providers reported knowing the $\mathrm{MgSO}_{4}$ guidelines "very well."

Guidelines and service protocols on EmONC were available in $12(52 \%)$ HCs and only seven (19\%) HPs. Over 50 percent of HC providers reported knowing the EmONC guidelines "very well," yet at the HP level a large percentage of HEWS said that no protocol for EmONC was available. Guidelines on safe and clean delivery were available in about half of the facilities (47\% of HPs and 57\% of HCs). Most HEWs reported knowing these guidelines "fairly well" (42\%) and "very well" (33\%), but 17 percent said there were no guidelines at their facilities. Most HC providers reported knowing the guidelines "very well" (85\%). Complete data on guideline availability, by facility type, are included in Appendix 8. 
Figure 3. Knowledge of guidelines for key maternal medicines

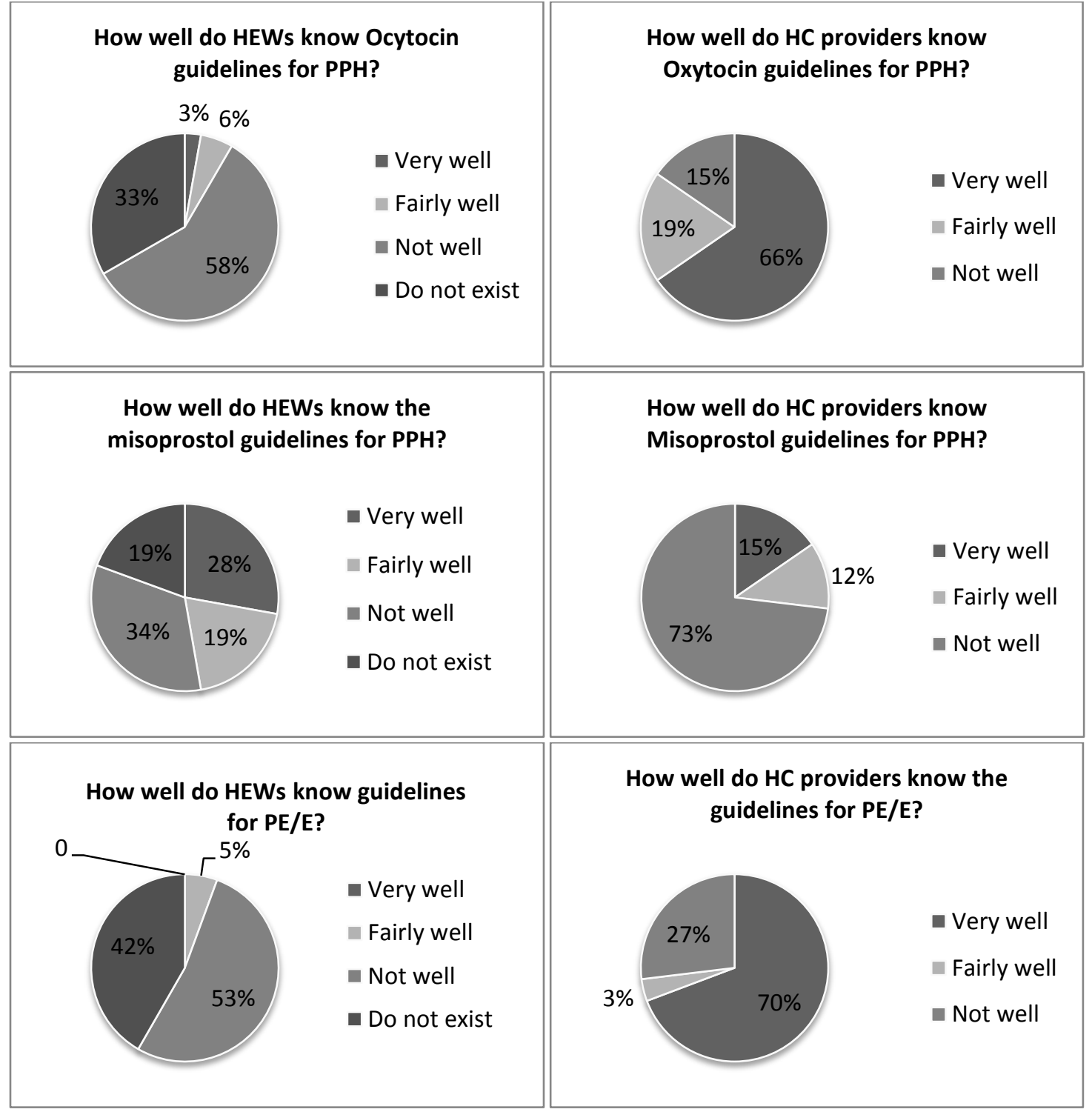

Source: Healthcare provider interview

\section{Provider knowledge, training, and experience}

All providers interviewed in HCs and HPs were aware that PPH is one of the five main causes of maternal mortality; most HC providers stated the other main causes, including PE/E (89\%), sepsis (96\%), obstructed labor $(80 \%)$, and unsafe abortion $(89 \%)$. HEWs were less informed. Oxytocin was recommended as the drug of choice for PPH by most HC providers (81\%), while 47 percent of HEWs identified misoprostol as the drug of choice for PPH (Figure 4). Knowledge about $\mathrm{MgSO}_{4}$ was limited among $\mathrm{HC}$ providers and HEWs (Figure 5). Data on provider knowledge, training, and experience are presented in Appendices 9 through 11. 
Figure 4. Knowledge of the drug of choice for the prevention and treatment of PPH

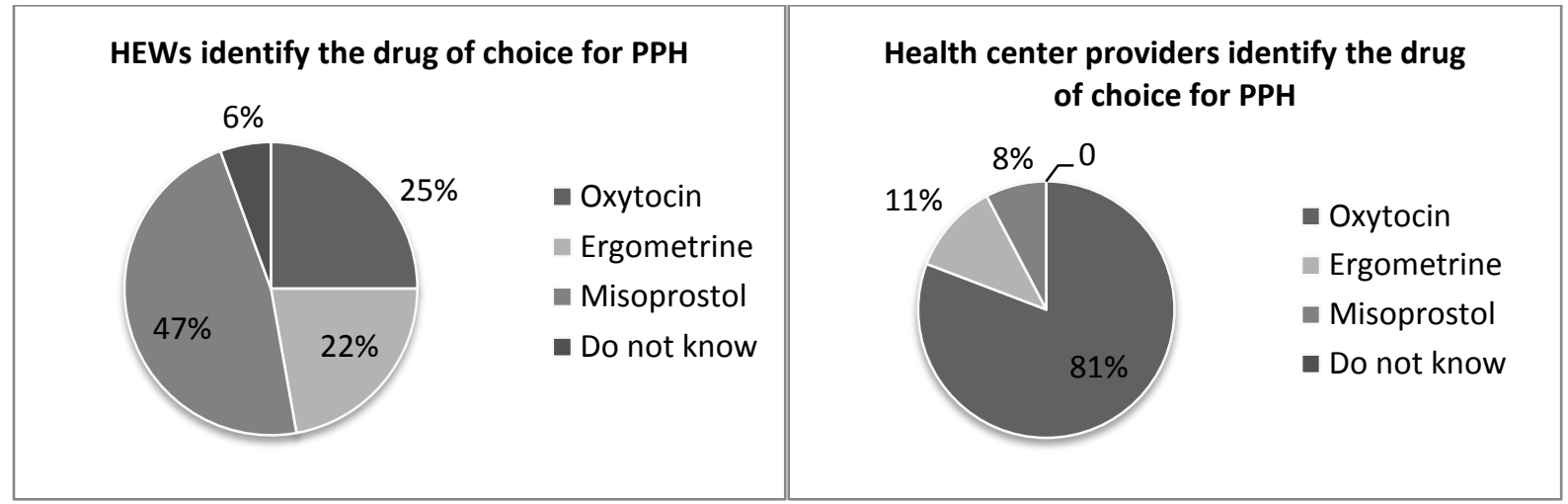

Source: Healthcare provider interview

Figure 5. Knowledge of the drug of choice for PE/E among health center providers

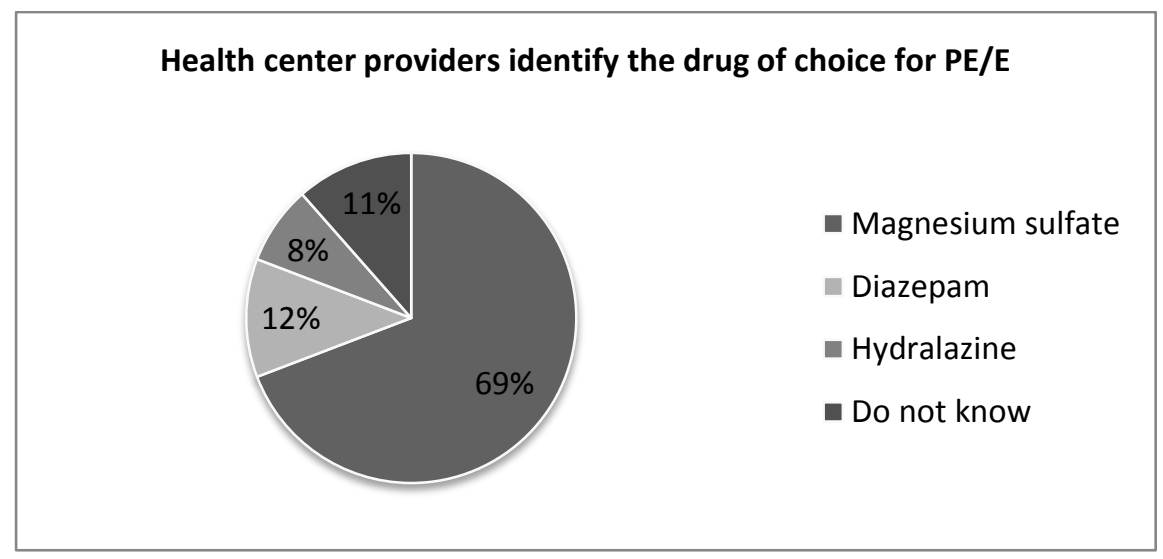

Source: Healthcare provider interview

Maternal health managers interviewed at zonal and district levels had insufficient awareness and knowledge of key maternal health supplies, particularly on the use of misoprostol for $\mathrm{PPH}$ and $\mathrm{MgSO}_{4}$ for $\mathrm{PE} / \mathrm{E}$. Many managers interviewed were not aware of the use of $\mathrm{MgSO}_{4}$ as the first line of treatment choice for PE/E, and most did not know whether misoprostol and $\mathrm{MgSO}_{4}$ were approved nationally. Furthermore, most did not know which health care providers were authorized to administer $\mathrm{MgSO}_{4}$ and misoprostol.

One maternal health officer indicated a lack of knowledge particularly about $\mathrm{MgSO}_{4}$ :

"With regard to $\mathrm{MgSO}_{4}$, frankly speaking, I never know this drug $\left[\mathrm{MgSO}_{4}\right]$; I was a little bit confused with another drug [magnesium trisilicate], which is commonly used for treating gastric problems ... As a professional and member of the woreda health office, we have never been discussing about $\left[\mathrm{MgSO}_{4}\right]$. Even it has never been mentioned by anyone of us in this office; we have never considered its role in the management of preeclampsia and eclampsia as other drugs such as diazepam. The woreda health office is expecting to support the health centers to ensure that life-saving drugs are available at the health facilities; however, I have never seen $\mathrm{MgSO}_{4}$ in our routine drug requisition and distribution at the health center or health post level. I have never heard it. Really, this drug is never been used in our facilities." 
A former health office head explained his gaps in knowledge about misoprostol:

"According to the information given by the Population Council, I think, HEWs are authorized to administer misoprostol at the health post level, but, I am not sure whether this is true at the national level. I know only the Population Council's misoprostol project, which was implemented in our woreda in few selected kebeles, but I do not know the policy directions and guidelines for misoprostol for PPH at the national level. Misoprostol is not included in our routine drug requisition and distribution and even not in the list of drugs for the health post. We do not receive this drug from the regional Pharmaceutical Fund and Supplies Agency [PFSA] and we have never requested this agency [PFSA] and the Bureau of health together other drugs during our routine drug request."

Roughly half of HEWs interviewed were aware that oxytocin (50\%) and misoprostol (53\%) are on Ethiopia's EDL; in contrast, only 27 percent of HC providers were aware misoprostol is included. Knowledge of national $\mathrm{MgSO}_{4}$ approval was poor among HEWS (8\%), and only 39 percent of HC providers were aware misoprostol is approved for PPH in Ethiopia. Furthermore, providers had limited knowledge of the guidelines for professional authorization of misoprostol administration in particular (Figure 6).

Figure 6: Knowledge of professional authorization

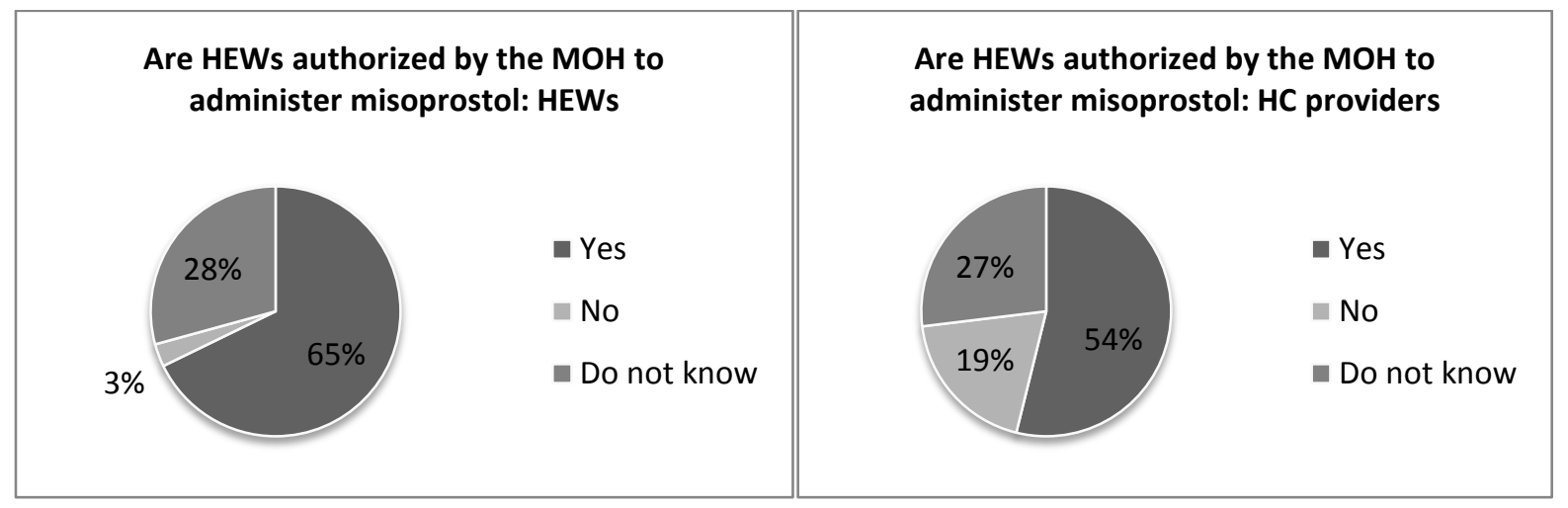

Source: Healthcare provider interview

One third of HEWs and all HC providers interviewed were trained, during their educations, on oxytocin, while smaller percentages of the two provider groups had refresher trainings in the last 12 months $(28 \%$ and $35 \%$, respectively). Significantly fewer providers received training in their professional education for misoprostol (11\% of HEWs and 39\% of HC providers); however, refresher training for HEWs on misoprostol was relatively high (47\%). For PE/E prevention and management, 22 percent of HEWs and 88 percent of HC providers received professional education and 17 percent and 27 percent received refresher training in the last year.

Nearly all providers received training on labor and delivery during their professional education $(89 \%$ of HEWs and $100 \%$ of HC providers), yet significantly fewer had refresher trainings in the last 12 months (58\% and $39 \%)$. All HC providers, and only five (14\%) HEWs also received training on complicated labor and birth during their professional training. Furthermore, only three (8\%) HEWS and 19 (73\%) HC providers reported training on EmONC during their professional education. Nearly all HC providers $(96 \%)$ and half of HEWs $(56 \%)$ received training on life-saving maternal health supplies during their professional education; refresher training in this subject was had by only 35 percent of HC providers and 53 percent of HEWs. 
Most HEWs (61\%) have administered misoprostol for PPH, compared to eight percent of HC providers. Nearly all HC providers (96\%) have performed AMTSL with oxytocin. Only 27 percent of HC workers have ever administered anticonvulsants for PE/E. HC providers' experience performing EmONC varied: 89 percent have performed manual removal of placenta, but fewer than 50 percent have ever performed assisted delivery, and only 12 percent have ever removed retained placenta with MVA. In qualitative interviews HC providers were asked to discuss their experiences in BEmONC. HC providers reported that most women with obstetric complications were referred to higher hospitals with better management available. Almost all providers reported that women with $\mathrm{PE} / \mathrm{E}$ were not managed at HCs because of fear of managing severe complications and lack of effective medicine, such as $\mathrm{MgSO}_{4}$. Lack of confidence, skills, and effective medicine were the main reasons stated for not managing women with obstetric complications in HCs.

\section{Supply chain management system}

With the support of the USAID/DELIVER project ${ }^{2}$, Ethiopia is currently implementing the new Integrated Pharmaceutical Logistic System (IPLS) under the leadership of the Pharmaceutical Fund and Supplies Agency (PFSA). The primary goal of the new IPLS is enabling facilities to prepare bimonthly commodity request (orders) to the PFSA hub warehouse that supports them (Figure 7). In this assessment, data were collected to assess the status of supply chain management system of the facilities, including inventory management of maternal health supplies and the level of implementation of the IPLS at HP and HC levels.

Figure 7: Overview of pharmaceuticals and information flow in the Integrated Pharmaceuticals Logistics Systems (IPLS)

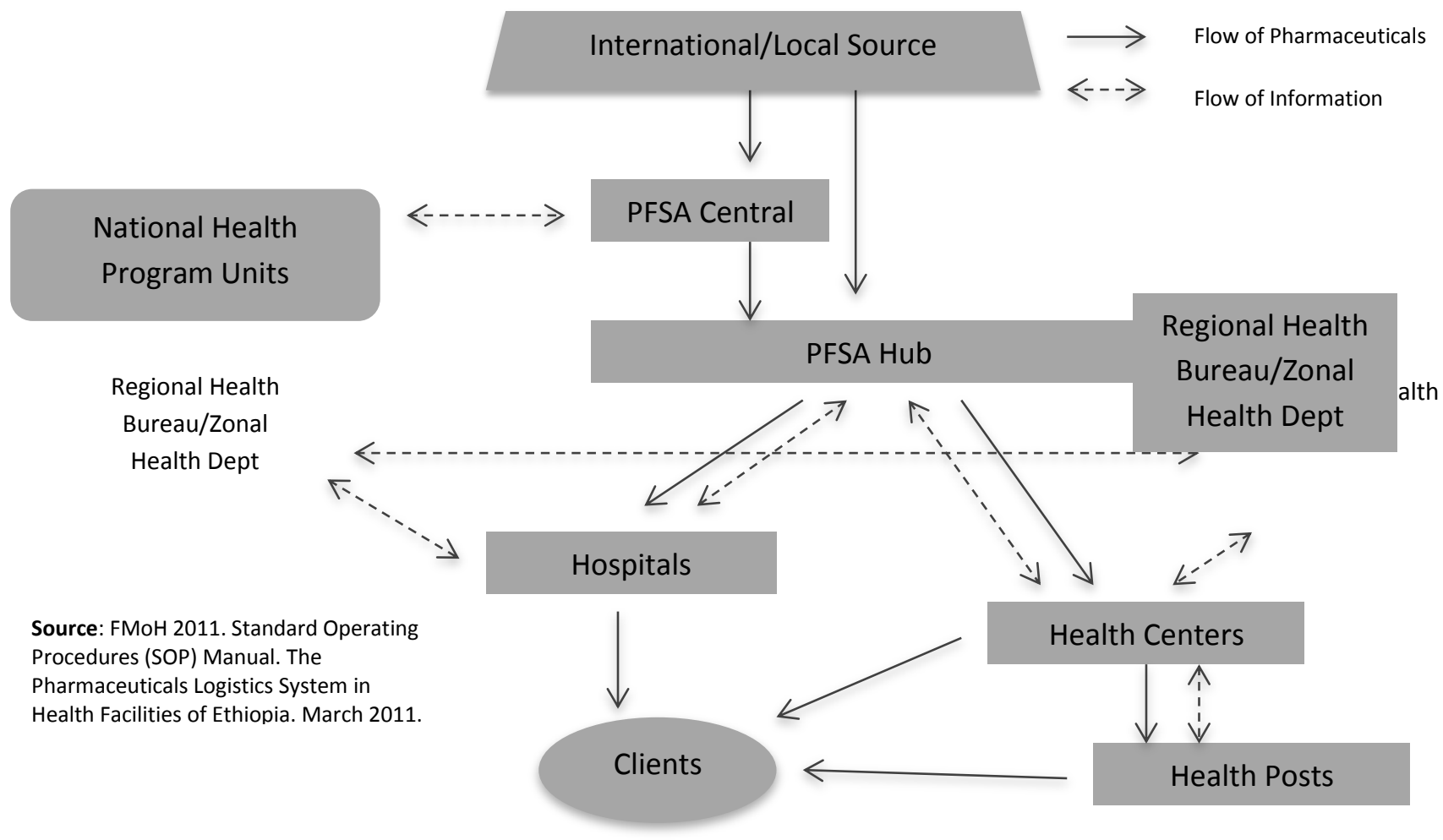

${ }^{2}$ http://deliver.jsi.com 
The present assessment indicates that 56 percent of HPs and 78 percent of HCs reported implementation of the new IPLS (Table 7). Only 28 percent of HPs and 13 percent of HCs, however, had stock cards for monitoring drug stock outs in their facilities. Written inventories of drugs were completed at only 22 percent of HPs and 44 percent of HCs in the 12 months prior to the assessment.

Table 7: Facility based supply chain management system conditions

\begin{tabular}{|l|c|c|}
\hline \multicolumn{1}{|c|}{ Supply chain management system conditions } & $\begin{array}{c}\text { Health post } \\
(\mathrm{n}=\mathbf{3 6})\end{array}$ & $\begin{array}{c}\text { Health center } \\
(\mathrm{n}=\mathbf{2 3})\end{array}$ \\
\hline Does the drug store have adequate space? & $\mathbf{2 3 ( 6 4 \% )}$ & $15(65 \%)$ \\
\hline Does the facility have adequate shelf for the drug? & $22(61 \%)$ & $12(52 \%)$ \\
\hline Is IPLS in place in this facility? & $20(55.6 \%)$ & $18(78 \%)$ \\
\hline Does this facility have stock card to monitor the status of drug stock-outs? & $10(28 \%)$ & $3(13 \%)$ \\
\hline Was written inventory made in this facility in the last 12 months? & $\mathbf{8 ( 2 2 \% )}$ & $10(44 \%)$ \\
\hline
\end{tabular}

Source: Inventory

\section{DISCUSSION}

\section{Availability of maternal health supplies}

Oxytocin is recommended for and readily available at HCs, with few stock outs, while misoprostol's availability is low in both HCs and HPs. Misoprostol is intended primarily for use in safe abortion at HCs, whereas in HPs it is intended for PPH prevention. Misoprostol for PPH is included on the national drug list and is recommended for administration by HEWs in HPs; furthermore, misoprostol has been successfully piloted in the study region. As a result, regular misoprostol supply should be prioritized, particularly for HPs.

$\mathrm{MgSO}_{4}$ was not available in either HCs or HPs, despite FMoH's approval and recommendation for its use in HCs. Additionally, anti-hypertensive drugs labetalol and Nefidipine, as well as calcium gluconate, an antidote for $\mathrm{MgSO}_{4}$ toxicity, were not available in HCs. The lack of availability of these key life-saving maternal medicines is a primary concern within the context of reducing maternal mortality due to $\mathrm{PE} / \mathrm{E}$ in Ethiopia.

Most supplies and materials needed for clean and safe delivery and EmONC services were available in HCs, but clean cloths for babies, in particular, were in short supply at both HCs and HPs. In addition, lack of availability of clean rooms, clean bed sheets, and adequate water at HPs remains a concern. Despite the fact that all HCs are designated to provide EmONC services, about one quarter of HCs did not have these services available and, in the 12 months prior to assessment, very few HCs reported performing all six or seven BEmONC signal functions.

\section{Needs, gaps, and challenges}

Given that oxytocin is largely available where intended for use, lessons from oxytocin can be applied to other key maternal medicines, namely misoprostol and $\mathrm{MgSO}_{4}$, which are relatively difficult to access in the health system. Factors contributing to oxytocin's availability include its continuous supply through IPLS, its incorporation within the system (rather than dependent on donor procurement and delivery), and HCs' ability to purchase it even with budget constraints. Although misoprostol and $\mathrm{MgSO}_{4}$ are approved for delivery by certain providers, they are not well integrated in IPLS; instead they are distributed mostly by international non-governmental organizations and largely dependent on donor funding. Qualitative interviews with providers and health managers reveal that lack of awareness and inadequate attention for misoprostol and $\mathrm{MgSO}_{4}$ use contribute to their lack of accessibility, while lack of clear $\mathrm{FMoH}$ guidance is another barrier. 
Overall, inadequate HP infrastructure (specifically lack of electricity and clean delivery rooms) continues to constrain HP and HEW capacity to provide clean and safe delivery services. In addition, at all levels, there is a need for more robust and reliable data collection of services provided and available equipment.

Finally, accurate and appropriate delivery of services by trained health providers is equally important as RH supply availability. Currently, lack of availability of well trained, knowledgeable, and experienced health providers at lower health system levels restricts the delivery of quality maternal and child health services. Nearly one fifth of the HPs and more than half of HCs reported health provider shortages. HC shortages occurred mostly among doctors, as well as health officers and nurses (with degrees). Overall knowledge of guidelines for maternal medicines was relatively poor and in need of improvement. Specifically, knowledge among HEWs of the five main maternal killers was weak, and provider knowledge of protocols for misoprostol and $\mathrm{MgSO}_{4}$ was limited. Training on guidelines and service protocols should be formally integrated into professional education programs of each cadre of healthcare provider at every level of the health system. Training programs should better emphasize EmONC services, delivery of oxytocin and misoprostol, and initial and refresher training on safe and clean delivery. All providers should be informed about which medicines are approved and recommended by the government and each provider should be knowledgeable about whether it is his/her responsibility to provide a specific medicine or intervention.

\section{Readiness for integrating misoprostol into HEP}

This assessment demonstrated that there is significant interest from HPs and HEWs for further integration of misoprostol in the Health Extension Program. To facilitate this goal, several key changes need to be made.

First, misoprostol must be integrated in to the national supply system. Misoprostol is currently not supplied by the government or integrated in to the national IPLS, which successfully supplies drugs like oxytocin. Instead, misoprostol relies on support from NGOs for its supply in HPs, which can be irregular. The majority of providers interviewed indicated that misoprostol had been supplied only once in their facilities and that it expired without refill in January 2013. Providers are unclear as to which organization provides misoprostol and therefore the process through which additional supply may be requested.

Second, providers indicated that there is insufficient training for HEWs on the delivery of misoprostol at the community level. There are no service protocols or guidelines for HEWs focused on misoprostol delivery at HPs. Furthermore, it is crucial that HC providers are also informed about misoprostol and its use in HPs, even if it is not indicated for HC use. Overall, there is a need for better understanding of the use of oxytocin and misoprostol together to prevent and treat PPH at different levels of the health system.

Finally, many health care providers also acknowledged that a lack of community awareness (and therefore demand), along with distances to facilities and lack of transportation are also challenges for access to maternal health supplies, including misoprostol in particular.

\section{Overall recommendations}

Ethiopia's pharmaceutical procurement and supply system is fragile and in need of further support. PFSA was established only six years ago, in 2007, and as a result, its IPLS is still evolving. The national system is intended to oversee quantification, procurement, and commodity distribution for the public sector. PFSA is financially independent and autonomous, and supplies regional nodes who then supply hospitals and woredas, who in turn supply HCs and HPs. Re-supply is normally monthly or bi-monthly, with emergency supply requests accepted ad hoc. Although this system has proved successful thus far, not all key medications (including misoprostol and $\mathrm{MgSO}_{4}$ ) are currently incorporated. 
Despite a burgeoning local pharmaceutical manufacturing industry in Ethiopia, there is an over-reliance on imported medications in addition to an over-reliance on the public sector for supply of key medicines. Although FMoH has recognized the importance of public and private partnerships, there is currently poor integration and understanding of the private sector's role in the supply and management of health supplies, medications, and equipment. In other countries the private sector has begun to utilize mobile and electronic health technologies to improve data tracking and collection for staffing, infrastructure, and services. Such technology is virtually nonexistent in Ethiopia; however, FHoH has developed a new eHealth strategy, which could be supported by public and private actors to ensure successful implementation (FMoH 2013).

Task shifting from 'skilled' providers to lower level HEWs has been a model of success in Ethiopia for a wide range of services. In-service training programs with job aids and checklists for Integrated Management of Neonatal and Childhood Illnesses (IMNCI) and Integrated Community Case Management (iCCM) pertaining to child and newborn health are in use, but job aids and checklists for $\mathrm{MNCH}$ medicines and services are under-developed, specifically for $\mathrm{MgSO}_{4}$ and misoprostol. Until recently, $\mathrm{MgSO}_{4}$ was employed for treatment of pre-eclampsia only in hospitals. $\mathrm{MgSO}_{4}$ is now recommended for use in $\mathrm{HCs}$, where most births occur, but decentralization of the intervention has yet to be successfully implemented. To further the process of decentralization to HCs, job aids and checklists for pre-eclampsia should also be supported. Incorporating refresher trainings on PPH prevention and treatment, treatment of pre-eclampsia, and safe and clean delivery to regular practice is essential for fully preparing providers at all levels of the system.

\section{Recommendations:}

\section{Increase availability of key maternal health medicines, supplies, and equipment:}

- Increase availability of $\mathrm{MgSO}_{4}$ and calcium gluconate at the $\mathrm{HC}$ level:

- Incorporate $\mathrm{MgSO}_{4}$ in to the national IPLS/PFSA;

- Increase availability of misoprostol at the HP level:

- Incorporate misoprostol in to the national IPLS/PFSA;

- Scale up misoprostol through mainstreaming into the Health Extension Program; this assessment indicates misoprostol was initiated both in home births and HPs, but not scaled up;

- Make available service delivery guidelines, protocols, and standards on misoprostol for PPH at the HC and HP levels; currently the Bureau of Health does not have these guidelines and protocols for misoprostol; lower level health managers have insufficient knowledge about it;

- Increase availability of supplies and the "six cleans" for clean and safe delivery at HPs;

- Increase availability of supplies and medical devices (such as MVA) for EmONC services.

\section{Increase awareness and knowledge of service provision guidelines for maternal health:}

- Further develop job aids and checklists for maternal and reproductive health on:

- The five main maternal killers (for HEWS);

- Misoprostol for prevention of PPH (for HEWs, HC providers and health managers);

- $\mathrm{MgSO}_{4}$ for treatment of PE/E (HC providers and health managers);

- At HPs and HCs, make a list of medicines approved and recommended for use available, specifying the system level at which the drug should be administered;

- Make service delivery guidelines, protocols, and standards on EmONC services available; 
- Increase demand for key maternal health interventions, supplies and medicines by improving community awareness.

\section{Expand existing training programs for HEWs and $\mathrm{HC}$ providers:}

- Improve routine refresher trainings for all $\mathrm{HC}$ providers on EmONC at the $\mathrm{HC}$ level;

- Improve routine refresher trainings for HEWs on safe and clean delivery for HEWs;

- Improve routine refresher trainings on key maternal health medicines for HC providers and HEWs.

4. Increase data collection and evaluation of service provision at the HC and HP level:

- Facilitate implementation of FMoH's eHealth strategy to establish workable m/eHealth systems;

- Build public and private partnerships to address challenges in supply chain management.

\section{Dissemination}

The findings of this assessment will be disseminated to policymakers, program managers and healthcare providers at all levels to discuss issues related to strengthening the primary healthcare system by improving the availability of life-saving maternal health supplies. In the dissemination workshop, special attention will be paid to the need to integrate misoprostol into the existing health extension program packages to ensure sustainability and regional scale up. The dissemination workshop may also serve as an advocacy strategy for creating commitment for misoprostol use to prevent PPH at the community level, where over 90 percent of births occur at home, without skilled birth attendants, as well as $\mathrm{MgSO}_{4}$ use and availability in $\mathrm{HCs}$. 


\section{APPENDICES}

Appendix 1: Complete list of facilities included in the assessment

1. Amba-Geworgis Health Center

2. Sak-Debir Health Post

3. Kosogie Health Post

4. Maksegnit Health Center

5. Work-Demo Health Post

6. Gedebiye Health Center

7. Abitira Health post

8. Wokin Health Cernter

9. Dil-Amba Health Post

10. Sertia health post

11. Chandiba Health Post

12. Chalia health post

13. Chilga Health Center

14. Chandiba Health Center

15. Sergauj Health Post

16. Minziro Health center

17. Hamsa-Fej Health post

18. Burbax Health post

19. Bahire-Ginb Health post

20. Jangua Health post

21. Chuhit Health Center

22. Koladiba Health Center

23. Salj Health post

24. Abrjaha Health center

25. Abrjaha health post

26. Darna Gawrna health post

27. Meskele-Crstos health post

28. Doromamay Health center

29. Amarit Health post

30. Felege-Hiwot health post

31. Amarit Health center

32. Adegoma health post

33. Arbaytu Ensesa health post

34. Aunt Menz health center

35. Rim Health Center

36. Giga health center

37. Zemene-Hiwot Health post

38. Birakat Health center

39. Tikur Bahir health post

40. Goshiye Health center

41. Debre-Mawi health center

42. Debre-mawi health post

43. Denbash health post

44. Lata Health center

45. Abiro Menor Health post

46. Wonjeta health post

47. Mercha health post

48. Mekene health post

49. Ambo mesk health post
50. Wogelsa heealth post

51. Sifatira health post

52. Mankusa Health center

53. Mana health post

54. Lata health post

55. Wojenta health center

56. Densa Bata health center

57. Dehna Mariam health post

58. Kudad health post

59. Wotet Abay Health center 
Appendix 2: Facility Infrastructure

\begin{tabular}{|l|c|c|}
\hline Facility infrastructure & Health Posts (36) & Health Centers (23) \\
\hline Rural & $36(100 \%)$ & $17(73.9 \%)$ \\
\hline Urban & $0(0 \%)$ & $6(26.1 \%)$ \\
\hline Piped Water & $3(8.3 \%)$ & $8(34.8 \%)$ \\
\hline Well water & $12(33.3 \%)$ & $3(13 \%)$ \\
\hline Electricity & $4(11.1 \%)$ & $18(78.3 \%)$ \\
\hline Landline telephone & $0(0 \%)$ & $12(52.2 \%)$ \\
\hline Mobile service & $33(91.7 \%)$ & $20(87 \%)$ \\
\hline Computer & $0(0 \%)$ & $18(78.3 \%)$ \\
\hline Toilet (working) & $28(77.8 \%)$ & $21(91.3 \%)$ \\
\hline Car in the facility & $0(0 \%)$ & $3(13 \%)$ \\
\hline Ambulance in the Woreda & $36(100 \%)$ & $23(100 \%)$ \\
\hline Clean delivery room & $16(44.4 \%)$ & $23(100 \%)$ \\
\hline
\end{tabular}

Appendix 3: Human Resource Availability

\begin{tabular}{|l|c|c|}
\hline Human Resources & Health Post (36) & Health Center (23) \\
\hline Medical Doctor/General practitioner) & N/A & $0(0 \%)$ \\
\hline OBGYN & N/A & N/A \\
\hline Health officer & N/A & $22(95.7 \%)$ \\
\hline Midwives & N/A & $22(95.7 \%)$ \\
\hline Nurses & N/A & $23(100 \%)$ \\
\hline Nurses with degree & N/A & $12(52.2 \%)$ \\
\hline Lab tech & O (0\%) & $22(95.7 \%)$ \\
\hline Pharm tech & N/A & $23(100 \%)$ \\
\hline Junior nurse & N/A & $6(26 \%)$ \\
\hline Health assistants & $36(100 \%)$ & $2(8.6 \%)$ \\
\hline Health extension workers & N/A & $10(43.5 \%)$ \\
\hline HMIS personnel & N/A & $18(78.3 \%)$ \\
\hline Healthcare provider shortage: Doctor & N/A & N/A \\
\hline Healthcare provider shortage: OBGYN & N/A & $13(56.5 \%)$ \\
\hline Healthcare provider shortage: Health officer & N/A & $13(56.5 \%)$ \\
\hline Healthcare provider shortage: Midwives & N/A & $12(52.2 \%)$ \\
\hline Healthcare provider shortage: Diploma nurse & N/A & $12(30.95 \%)$ \\
\hline Healthcare provider shortage: Degree nurse & $10.4 \%)$ & N/A \\
\hline Healthcare provider shortage: Lab tech & $10(43.5 \%)$ \\
\hline Healthcare provider shortage: Pharm tech & N/A & \\
\hline Shortage of HEWs & N & $10 \%)$ \\
\hline
\end{tabular}


Appendix 4: Availability of equipment, materials and medical devices for safe and clean delivery and EmONC

\begin{tabular}{|c|c|c|}
\hline $\begin{array}{l}\text { Type of equipment, material/medical } \\
\text { device }\end{array}$ & Health post $(n=36)$ & Health center $(n=23)$ \\
\hline Torch & $4(11.1 \%)$ & $11(47.8 \%)$ \\
\hline BP apparatus & $30(83.3 \%)$ & $22(95.7 \%)$ \\
\hline Delivery set (complete) & $33(91.7 \%)$ & $21(91.3 \%)$ \\
\hline Stetescope & $32(88.9 \%)$ & $23(100 \%)$ \\
\hline Refrigerator & $9(25 \%)$ & $23(100 \%)$ \\
\hline Examination couch & $10(27.8 \%)$ & $23(100 \%)$ \\
\hline Delivery couch & $31(86.1 \%)$ & $23(100 \%)$ \\
\hline Ambubag (resuscitation device) & $12(33.3 \%)$ & $10(43.5 \%)$ \\
\hline Valve syringe (resuscitation device) & $6(16.7 \%)$ & $20(87 \%)$ \\
\hline Apron & $30(83.3 \%)$ & $20(87 \%)$ \\
\hline Screen & $5(13.9 \%)$ & $21(91.3 \%)$ \\
\hline Baby scale & $26(72.2 \%)$ & $15(65.5 \%)$ \\
\hline Adult scale & $27(75 \%)$ & $23(100 \%)$ \\
\hline Fetoscope & $34(94.4 \%)$ & $22(95.7 \%)$ \\
\hline Tape meter & $23(63.9 \%)$ & $14(60.9 \%)$ \\
\hline Disposable glove & $25(69.4 \%)$ & $17(73.9 \%)$ \\
\hline Surgical glove & $27(75 \%)$ & $19(82.6 \%)$ \\
\hline Thermometer (oral) & 4 (11.1\%) & $10(4.3 \%)$ \\
\hline Thermometer (axillary ) & $26(72.2 \%)$ & $16(69.6 \%)$ \\
\hline Thermometer (rectal) & $10(2.8 \%)$ & $10(4.3 \%)$ \\
\hline Syringe and needle & $32(88.9 \%)$ & $22(95.7 \%)$ \\
\hline Speculum & $9(27.3 \%)$ & $10(43.5 \%)$ \\
\hline Cannula & $7(19.4 \%)$ & $6(26.1 \%)$ \\
\hline Sponge forceps & $12(33.3 \%)$ & $12(52.2 \%)$ \\
\hline Artery forceps & $24(66.7)$ & $17(73.9 \%)$ \\
\hline Manual vacuum aspiration (MVA) & N/A & $11(47.8 \%)$ \\
\hline D \&C set & N/A & N/A \\
\hline Kidney dish & $33(91.7 \%)$ & $21(91.3 \%)$ \\
\hline Cut Gut (suturing material) & $7(19.4 \%)$ & $6(26.1 \%)$ \\
\hline Scissors & $31(86.1 \%)$ & $21(91.3 \%)$ \\
\hline Cotton roll & $31(86.1 \%)$ & $20(87 \%)$ \\
\hline Microscope & $\mathrm{N} / \mathrm{A}$ & $23(100 \%)$ \\
\hline \multicolumn{3}{|l|}{ Disinfectant/Antiseptic solutions } \\
\hline Savlon & $6(16.7 \%)$ & $13(56.5 \%)$ \\
\hline lodine & $14(39.9 \%)$ & $16(69.6 \%)$ \\
\hline Alcohol (70\%) & $6(16.7 \%)$ & $13(56.5 \%)$ \\
\hline
\end{tabular}


Appendix 5: Maternal Medicines

\begin{tabular}{|l|c|c|}
\hline Maternal Medicines & Health Post (36) & Health Center (23) \\
\hline Oxytocin & N/A & $23(100 \%)$ \\
\hline Misoprostol & $2(5.6 \%)$ & $9(39.1 \%)$ \\
\hline Ergometrine & N/A & $17(73.9 \%)$ \\
\hline MgS04 & N/A & $0(0 \%)$ \\
\hline Calcium gluconate & N/A & $1(4.3 \%)$ \\
\hline Diazepam & $1(2.8 \%)$ & $16(69.6 \%)$ \\
\hline Hydralizine & $1(2.8 \%)$ & $13(56.5 \%)$ \\
\hline Nefidipine & N/A & $8(34.8 \%)$ \\
\hline Methyldopa & N/A & $18(78.3 \%)$ \\
\hline Labetalol & N/A & $0(0 \%)$ \\
\hline Ampicillin injection & N/A & $19(82.6 \%)$ \\
\hline Ampicillin capsule & N/A & $23(100 \%)$ \\
\hline Metronidazole injection & N/A & $1(4.3 \%)$ \\
\hline Gentamycin & $0(0 \%)$ & $23(100 \%)$ \\
\hline Folic acid & $1(2.8 \%)$ & $5(21.7 \%)$ \\
\hline Ferous sulfate & $5(13.9 \%)$ & $10(43.5 \%)$ \\
\hline Vitamin A & $31(86 \%)$ & $17(74 \%)$ \\
\hline Normal saline & N/A & $22(95.7 \%)$ \\
\hline Ringer lactate & N/A & $20(87 \%)$ \\
\hline Glucose & $1(2.8 \%)$ & $22(95.7 \%)$ \\
\hline Adrenaline & N/A & $23(100 \%)$ \\
\hline Erythromaycin & $11(47.8 \%)$ \\
\hline Tetracycline & & \\
\hline
\end{tabular}

Appendix 6: Contraceptive availability

\begin{tabular}{|l|c|c|}
\hline Contraceptives & Health Post (36) & Health Center (23) \\
\hline Depo-provera & $34(94.4 \%)$ & $23(100 \%)$ \\
\hline Combined pills & $23(63.9 \%)$ & $17(73.9 \%)$ \\
\hline Projesterone only pills & $1(2.8 \%)$ & $20(87 \%)$ \\
\hline Emergency pills & $5(13.9 \%)$ & $11(47.8 \%)$ \\
\hline Jadelle & $3(8.3 \%)$ & $11(47.8 \%)$ \\
\hline Implanon & $18(50 \%)$ & $14(60.9 \%)$ \\
\hline IUCD & $3(8.3 \%)$ & $9(39.1 \%)$ \\
\hline Male condom & $26(72.2 \%)$ & $22(95.7 \%)$ \\
\hline Female condom & $0(0 \%)$ & $0(0 \%)$ \\
\hline
\end{tabular}

*Note: Norplant was not included in the survey. 
Appendix 7: Expansion and scale-up of misoprostol for PPH

Misoprostol piloted at health post level: HEWs' perspective

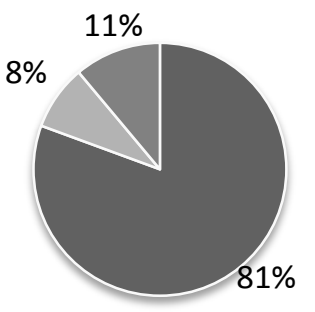

Misoprostol piloted at health post level: HC providers' perspective

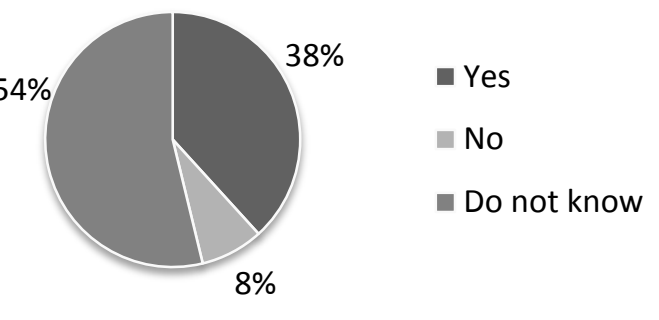

Misoprostol scaled-up at health post level: HEWs' perspective

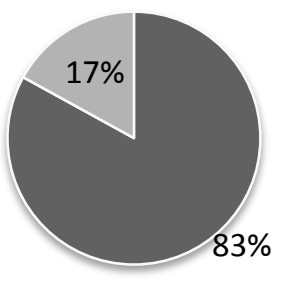

Yes

No

Do not know
Misoprostol scaled-up at health post level: HC providers' perspective

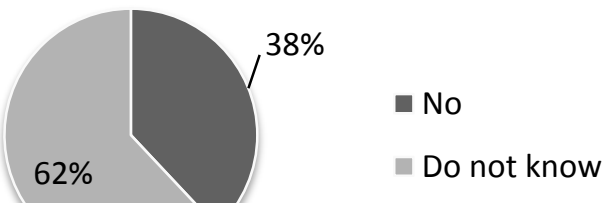

Appendix 8: Availability of guidelines/service protocols for maternal health care services

\begin{tabular}{|l|c|c|}
\hline Guidelines/Service Protocols & Health Post (36) & Health Center (23) \\
\hline Prevention of PPH with oxytocin & $2(5.5 \%)$ & $11(47.8 \%)$ \\
\hline Treatment of PPH with misoprostol & $5(13.8 \%)$ & $4(17.4 \%)$ \\
\hline Prevention/treatment of PE/E & $3(8.3 \%)$ & $11(47.8 \%)$ \\
\hline Protocol for FP services & $21(58.3 \%)$ & $18(78.3 \%)$ \\
\hline Protocol for EmONC services & $7(19.4 \%)$ & $12(52.2 \%)$ \\
\hline Protocol for Focused ANC services & $23(63.8 \%)$ & $17(73.9 \%)$ \\
\hline Protocol for PNC services & $21(58.3 \%)$ & $14(60.9 \%)$ \\
\hline Protocol for clean and safe delivery services & $17(47.2 \%)$ & $13(56.5 \%)$ \\
\hline Protocol for infection prevention/universal precaution & $11(30.5 \%)$ & $12(52.2 \%)$ \\
\hline
\end{tabular}


Appendix 9: Provider Knowledge

\begin{tabular}{|l|c|c|}
\hline Provider Knowledge & Health Post (36) & Health Center (26) \\
\hline Hemorrhage is one of the 5 main killers & $36(100 \%)$ & $26(100 \%)$ \\
\hline PE/E is one of the 5 main killers & $22(61.1 \%)$ & $23(88.5 \%)$ \\
\hline Sepsis is one of the 5 main killers & $16(44.4 \%)$ & $25(96.2 \%)$ \\
\hline Obstructed labor is one of the 5 main killers & $19(52.8 \%)$ & $21(80.8 \%)$ \\
\hline Unsafe abortion is one of the 5 main killers & $17(47.2 \%)$ & $23(88.5 \%)$ \\
\hline Is Oxytocin on the EDL of the country? & $18(50 \%)$ & $24(92.3 \%)$ \\
\hline Is misoprostol on EDL of the country? & $19(52.8 \%)$ & $7(26.9 \%)$ \\
\hline Is MgSO4 on EDL of the country? & $6(16.7 \%)$ & $19(73.1 \%)$ \\
\hline Is MgSO4 in the national policy of PE/E? & $4(11.1 \%)$ & $16(61.5 \%)$ \\
\hline Is MgSO4 approved by the country for PE/E? & $3(8.3 \%)$ & $19(73.1 \%)$ \\
\hline Is misoprostol approved by the country for PPH? & $23(63.9 \%)$ & $10(38.5 \%)$ \\
\hline Are midwives authorized to perform AMTSL with oxytocin? & $24(66.7 \%)$ & $26(100 \%)$ \\
\hline Are HEWs authorized to administer misoprostol for PPH? & $25(69.4 \%)$ & $14(53.8 \%)$ \\
\hline
\end{tabular}


Appendix 10: Provider training

\begin{tabular}{|c|c|c|}
\hline Provider Training & $\begin{array}{l}\text { Health Post } \\
(36)\end{array}$ & $\begin{array}{l}\text { Health Center } \\
\text { (26) }\end{array}$ \\
\hline Training on prevention/management of PPH with oxytocin during education & $12(33.3 \%)$ & $26(100 \%)$ \\
\hline $\begin{array}{l}\text { Refresher training on prevention/management of PPH with oxytocin during last } 12 \\
\text { months? }\end{array}$ & $10(27.8 \%)$ & $9(34.6 \%)$ \\
\hline $\begin{array}{l}\text { Received training on prevention and management of PPH with misoprostol during } \\
\text { education? }\end{array}$ & $4(11.1 \%)$ & $10(38.5 \%)$ \\
\hline Refresher training on misoprostol in last 12 months? & $17(47.2 \%)$ & $5(19.2 \%)$ \\
\hline Training on prevention and management of severe $\mathrm{PE} / \mathrm{E}$ during education? & $8(22.2 \%)$ & $23(88.5 \%)$ \\
\hline Refresher training on PE/E? & $6(16.7 \%)$ & $7(26.9 \%)$ \\
\hline Training on normal delivery during education & $33(91.7 \%)$ & $26(100 \%)$ \\
\hline Refresher training on normal delivery during last 12 months & $23(63.9 \%)$ & $10(38.5 \%)$ \\
\hline Training on complicated labor and birth during education? & $5(13.9 \%)$ & $26(100 \%)$ \\
\hline Training on complicated birth in the last 12 months? & $5(13.9 \%)$ & 9 (34.6\%) \\
\hline $\begin{array}{l}\text { Training on life-saving maternal health supplies during pregnancy/childbirth or after } \\
\text { delivery during professional education? }\end{array}$ & $20(55.6 \%)$ & $25(96.2 \%)$ \\
\hline $\begin{array}{l}\text { Refresher training on life-saving maternal health supplies during pregnancy/childbirth } \\
\text { or after delivery during last } 12 \text { months? }\end{array}$ & $19(52.8 \%)$ & $9(34.6 \%)$ \\
\hline Training on EmONC during professional education? & $3(8.3 \%)$ & $19(73.1 \%)$ \\
\hline Refresher training on EmONC in last 12 months? & $5(13.9 \%)$ & $7(26.9 \%)$ \\
\hline Training on safe and clean delivery during professional education? & $32(88.9 \%)$ & $26(100 \%)$ \\
\hline Refresher on safe and clean delivery in last 12 months? & $21(58.3 \%)$ & $10(38.5 \%)$ \\
\hline
\end{tabular}

Appendix 11: Provider experience

\begin{tabular}{|l|c|c|}
\hline Provider Experience & Health Post (36) & Health Center (26) \\
\hline Ever performed AMTSL with oxytocin & N/A & $25(96.2 \%)$ \\
\hline Ever administer misoprostol for PPH & $22(61.1 \%)$ & $2(7.7 \%)$ \\
\hline Ever administered parenteral anticonvulsant drugs for PE/E & N/A & $7(26.9 \%)$ \\
\hline Ever administered parenteral antibiotics for maternal sepsis & N/A & $16(61.5 \%)$ \\
\hline Ever performed manual removal of placenta & N/A & $23(88.5 \%)$ \\
\hline Ever performed removal of retained placenta with MVA & N/A & $3(11.5 \%)$ \\
\hline Ever performed assisted delivery & N/A & $11(42.3 \%)$ \\
\hline
\end{tabular}




\section{REFERENCES}

Carrolin, G., C. Cuesta, E. Abalos and A.M. Gulmezoglu. 2008. Epidemiology of postpartum hemorrhage: a systematic review. Best Practice \& Research Clinical Obstetrics and Gynecology 22: 999-1012.

Central Statistical Agency (CSA), ORC Macro: Ethiopia 2011 Demographic and Heath Survey. Addis Ababa, Ethiopia

Ethiopia Demographic and Health Survey (EDHS). 2011. Central Statistical Agency and ORC Macro. Addis Ababa and Calverton, Maryland, USA: ICF International.

Ethiopia Demographic and Health Survey. 2005. Central Statistical Agency and ORC Macro. Addis Ababa and Calverton, Maryland, USA: ICF International.

Fernandez, M.M., F. Coeytaux, R.G. de León and D.L. Harrison. 2009. Assessing the global availability of misoprostol. The International Journal of Gynecology \& Obstetrics 105(2): 180-186.

Food, Medicine and Healthcare Administration and Control Authority of Ethiopia. List of Essential Medicines for Ethiopia, Fourth Edition. Addis Ababa, September 2010. http://apps.who.int/medicinedocs/documents/s17568en/s17568en.pdf

Federal Ministry of Health (FMoH). 2010. Health Sector Development Programme IV 2010-11-2014-15: www.internationalhealthpartnership.net/fileadmin/uploads/ihp/Documents/Country Pages/ Ethiopia/Ethiopia HSDP IV Final \%202010\%20-2015.pdf

Federal Ministry of Health $(\mathrm{FMoH}), 2011$. Standard Operating Procedures (SoP) Manual. The Pharmaceuticals Logistics System in Health Facilities of Ethiopia. March 2011.

Federal Ministry of Health (FMoH). 2012. Road map for accelerating the reduction of maternal and newborn morbidity and mortality in Ethiopia: 2012-2015.

Federal Ministry of Health (FMoH), 2013. Ethiopian National eHealth Strategy 2013-2015. Draft, version 2.

Hofmeyr, G.J., A.M. Gülmezoglu, N. Novikova and T.A. Lawrie. 2013. Postpartum misoprostol for preventing maternal mortality and morbidity. Cochrane Database of Systematic Reviews 7. Art.: CD008982. DOI: 10.1002/14651858.CD008982.pub2.

Hogan, M.C., K.J. Foreman, M.M. Naghavi, S.Y. Ahn, M. Wang, S.M. Makela, A.D. Lopez, R. Lozano and C.J. Murray. 2010. Maternal mortality for 181 countries, 1980-2008: A systematic analysis of progress towards Millennium Development Goal 5. The Lancet. 375(9726): 1609-1623

Khan, K., D. Wojdyla, L. Say, A.M. Gulmezoglu and P. Van Look. 2006. Analysis of causes of maternal deaths: a systematic review. The Lancet 367: 1066-1074.

Koblinsky, M., F. Tain and S. Tesfaye. 2010. Reducing maternal mortality and increasing use of skilled birth attendance: Ethiopia and MDG 5. Ethiopian Journal of Reproductive Health 4(1): 4-10.

Maine, D. and S. Thaddeus. 1994. Too far to Walk: maternal mortality in context. Soc Sci Med 38(8): 10911100 .

Management Sciences for Health (MSH), WHO. International Drug Price Indicator Guide. 2010 ed.; 2011.

Morrison, J.S. and S. Brundage. 2012. Advancing Health in Ethiopia With Fewer Resources, an Uncertain GHI Strategy, and Vulnerabilities on the Ground. A report of the CSIS Global Health Policy Center.

POPPHI. 2007. Prevention of Postpartum Hemorrhage: Implementing Active. Management of the Third Stage of Labor (AMTSL): A Reference Manual for Health Care Providers. Seattle: PATH.

POPPHI. 2008. Use of oxytocin and misoprostol for induction or augmentation of labor in low-resource settings. A working paper review for POPPHI project, PATH, Washington, D.C. Prepared by: Ann Lovold and Cynthia Stanton. March 2008. 
PATH. 2008. Uterotonic Drugs for the Prevention and Treatment of Postpartum Hemorrhage [factsheet]: www.path.org/publications/files/MCHN_popphi_pph_fs_uterotonic.pdf. Seattle: PATH. Accessed February 7, 2012.

Raifman, S., S. Melese, K. Hailemariam, G. Kassa, A. Shiferaw and A. Erulkar. 2012. Increasing Access to Family Planning and Reproductive Health Through Community Health Workers: A Case Study of a Dual Cadre Model in Rural Ethiopia. Washington, D.C.: Population Council.

Smith, J.M., R.F. Lowe, J. Fullerton, S.M. Currie, S. Harris and E. Felker-Kantor. 2013. An integrative review of the side effects related to the use of $\mathrm{MgSO} 4$ for pre-eclampsia and eclampsia management. BMC Pregnancy and Childbirth 13: 34.

UN Commission Report. 2012. UN Commission on life-saving commodities for women and children: www.unfpa.org/webdav/site/global/shared/images/publications/2012/Final $\% 20$ UN $\% 20$ Commission $\% 20 \mathrm{R}$ eport 14sept2012.pdf

UNFPA. 2008. Summary and statistical report of the 2007 Population and Housing Census. Population size by age and sex. Addis Ababa: Population Census Commission, Federal Democratic Republic of Ethiopia.

UNICEF, WHO, UNFPA. 1997. Guidelines for monitoring the availability and use of obstetric services.

UNICEF and FMoH Safe Motherhood Technical Working Group. 2004. Safe and Clean Birth and Newborn Care: A Reference for Health Extension Workers. Adapted from A Book for Midwives: Care for Pregnancy, Birth and Women's Health by S. Klein, S. Miller and F. Thompson (Hesperian Foundation, Berkeley, 2004).

World Health Organization (WHO). 2009. WHO statement regarding the use of misoprostol for postpartum hemorrhage prevention and treatment. Geneva: WHO.

World Health Organization (WHO). 2011. WHO recommendation for preventing and treatment of preeclampsia and eclampsia. Geneva: WHO.

World Health Organization (WHO). 2008. Education materials for teachers of midwifery: midwifery education modules, second edition. Managing puerperal sepsis. Geneva: WHO. http://whqlibdoc.who.int/publications/2008/9789241546669 6 eng.pdf 

